\title{
Influence of Schistosoma japonicum programmed cell death protein 10 on the growth and development of schistosomula
}

\author{
Yan Ru Gao ${ }^{1,2,3}$, Wen Ling Huang ${ }^{1}$, Chun Lian Tang ${ }^{4}$, Rong Liu', Qin Ping Zhao', Zhen Ping Ming ${ }^{1}$
} and Hui Fen Dong ${ }^{1 *}$

\begin{abstract}
Background: Schistosomiasis caused by Schistosoma japonicum is among the most serious endemic zoonoses in China. To study interactions between schistosomula, the pre-adult juvenile stage, and hosts, it is important to study the functions of key genes involved in schistosomula growth and development. Programmed cell death protein 10 ( $p c d p 10)$ is an important apoptosis-related gene with various biological functions. This study described the molecular characterization of S. japonicum PCDP10 (SJPCDP10) and evaluated its functions in schistosomula.
\end{abstract}

Methods: Real-time quantitative polymerase chain reaction (qPCR) and western blot were used to detect Sjpcdp10 mRNA and protein levels, respectively, at different developmental stages. Immunolocalization was performed to determine SjPCDP10 expression in the parasite. RNA interference (RNAi) experiments were used to assess gene functions associated with SjPCDP10 in schistosomula growth and development.

Results: Real-time qPCR revealed that Sjpcdp10 was expressed during all investigated developmental stages and upregulated during schistosomula growth and development. Histochemical localization showed that SJPCDP10 was mainly distributed in the teguments of schistosomula in all investigated stages and part of the parenchymal area of 14-, 18-, and 21-day-old schistosomula. Following Sjpcdp10 knockdown by RNAi, the lengths, widths, areas, and volumes of schistosomula were significantly lower than those in the control group. Scanning electron microscopy showed that the body surfaces of schistosomula subjected to RNAi were seriously damaged, with few tegumental spines and sensory papillae. Transmission electron microscopy indicated that the teguments of Sjpcdp10-knockdown schistosomula were incomplete, the number of layers was reduced, and the thickness decreased significantly as compared with those in the control group. Furthermore, terminal deoxynucleotidyl transferase dUTP nick-end labelling results showed that the rate of apoptosis in Sjpcdp10-knockdown schistosomula was significantly higher than that in the control group.

Conclusions: Sjpcdp10-knockdown influenced the growth and development of schistosomula. Therefore, our results indicated that SjPCDP10 contributes to the regulation of cell apoptosis and is essential for schistosomula growth and development.

Keywords: Schistosoma japonicum, Schistosomula, Programmed cell death factor-10, Growth and development, Tegument, Apoptosis

\footnotetext{
* Correspondence: hfdong@whu.edu.cn

${ }^{1}$ Hubei Province Key Laboratory of Allergy and Immunology, Department of

Parasitology, Wuhan University School of Basic Medical Sciences, Wuhan,

Hubei Province 430071, China

Full list of author information is available at the end of the article
} 


\section{Background}

Schistosomiasis is a "neglected tropical disease" that affects over 200 million people in 78 countries and is considered a chronic and poverty-promoting disease [1]. Schistosoma japonicum is the only human blood fluke that occurs in China. As of 2015, there were > 30, 000 villages (containing 68 million residents) endemic for schistosomiasis [2]. As one of the countries routinely affected by epidemic diseases, China has made great strides in schistosomiasis. However, there are emerging challenges, including limitations in new drug development and the lack of an available vaccine [3-6]. Although some vaccines have been developed, the ideal immunity rate has not been achieved [5]. One reason for this might be that growth and developmental mechanisms and its interactions with the host are poorly understood [7]. To this end, the study of schistosomulumspecific molecules is not only important for identifying new functional genes as potential vaccine antigens or drug targets for human schistosomiasis but also helpful in revealing mechanisms of growth, development, and interactions with the host. Therefore, it is necessary to study the functions of the key genes involved in S. japonicum growth and development in more detail.

Studies of schistosomulum staging reported that inhibiting the expression of cathepsin B in Schistosoma mansoni might lead to schistosomula growth retardation [8]. Furthermore, RNAi-mediated knockdown of the 26S proteasome subunit SmRPN11/POH1 affects schistosomula development and survival [9]. Our laboratory screened for genes, such as orthologues of adenylate kinase 1, programmed cell death protein 10 (pcdp10), and heat-shock protein 70 , that might play an important role in the growth and development of S. japonicum schistosomula [10, 11]. Among these, PCDP10 is an important molecule involved in apoptosis regulation and was originally named TF-1 cell apoptosis-related factor15 based on its cloning from human erythroleukemia TF-1 cell lines [12]. Functional studies have shown that PCDP10 exhibits a variety of biological functions in human cells, including inhibiting tumour-cell apoptosis and promoting blood-vessel regeneration and reconstruction [13, 14]. Although there are many reports regarding the function of human PCDP10, few focused on parasite PCDP10 proteins, including that of S. japonicum (SjPCDP10). Sjpcdp10 was also found to be differentially expressed between schistosomula cultured in vitro and those derived from hosts [10], and it was hypothesized that SjPCDP10 might be involved in schistosomula growth and development. Therefore, in this study, the sequence characteristics, localization, and expression levels of SjPCDP10 were analyzed throughout the life-cycle of S. japonicum. Our results showed that RNA-mediated Sjpcdp10 knockdown resulted in severe morphological damage. These results deepen our understanding of the biological role of this gene in S. japonicum development.

\section{Methods}

\section{Animals and parasites}

Female Kunming mice (6-8 week-old, 20-25 g each) were purchased from Wuhan University Laboratory Animal Centre and randomly divided into six groups. Two adult male New Zealand rabbits (2.5 kg each) and Oncomelania hupensis snails infected with S. japonicum were purchased from Hubei Provincial Center for Disease Control and Prevention. Cercariae were collected routinely by exposing $O$. hupensis snails in water to light for 3 to $4 \mathrm{~h}$ to induce parasite shedding. Sixty mice were divided into six groups and infected with different numbers of cercariae (8000, 5000, 800, 600,200 and 100 cercariae) via abdominal-skin exposure. After infection, mice were killed and the schistosomulae were collected at different time points.

(30 $\mathrm{min}, 3$ days, 10 days, 14 days, 18 days and 21 days after infection). For skin-type schistosomula (30 min), infected skin was directly removed, cut into pieces and then incubated with $10 \mathrm{ml}$ phosphate-buffered saline (PBS) at $37{ }^{\circ} \mathrm{C}$ for $2 \mathrm{~h}$. The incubated mixture was filtered through a filter screen (140-mesh) (Wuhan Kerui Biological Technology Co. Ltd., Wuhan, China) [10]. The other schistosomula were collected by perfusion of mice according to methods previously described [15]. All experimental data were derived from in vivo living schistosomula except the schistosomulae of RNAi experiments, which was mechanically transformed by cercariae in vitro.

\section{Molecular cloning and sequence analysis of SjPCDP10}

Full-length complementary DNA (cDNA) was obtained using reverse transcription polymerase chain reaction (RT-PCR) with mRNA template prepared from lungstage schistosomula (3 days after infection). Primers were designed by Oligo 6 software (http://www.oligo.net/) per the Sjpcdp10 mRNA sequence (accession no. FN326945.1) obtained from GenBank. Forward (5' -CCG GAA TTC ATG GCT GGA AGT AAG TGG C-3') and reverse (5'-CCG CTC GAG ATC CAC ATC GTG AAC C-3') primers containing EcoRI and Xhol restriction sites were used to amplify the target gene by PCR according the following amplification protocol: $95^{\circ} \mathrm{C}$ for $5 \mathrm{~min} ; 35$ cycles at $95{ }^{\circ} \mathrm{C}$ for $30 \mathrm{~s}, 58{ }^{\circ} \mathrm{C}$ for $30 \mathrm{~s}$, and $72{ }^{\circ} \mathrm{C}$ for $90 \mathrm{~s}$, followed by a final extension at $72{ }^{\circ} \mathrm{C}$ for $8 \mathrm{~min}$. The PCR products were purified using AxyPrep DNA gel extraction kit (Axygen, Hangzhou, China) according to the standard protocol and cloned into the prokaryotic expression vector pET28a (+) (Novagen, Madison, USA). Recombinant Sjpcdp10 (rSjpcdp10) 
plasmids were transformed into competent Escherichia coli DH5 $\alpha$ cells, and positive clones were screened and identified by PCR, enzyme analysis, and sequencing (Sunny Biotechnology Co., Ltd., Shanghai, China).

The molecular characteristics of SjPCDP10 were analyzed using a variety of bioinformatics approaches. The amino acid sequence was used as a query to identify PCDP10 orthologues. An alignment of protein sequences exhibiting sufficient similarity from different species was generated by ClusalX 2.0 (http://www.clustal.org/clustal2/). A phylogenetic tree was generated using the neighbour-joining method by MEGA5.05 software (The Biodesign Institute, Tempe, AZ, USA) to analyze relationships between SjPCDP10 and PCDP10 homologs from other species. The stability of the amino acid sequences was predicted by ProtParam (http://web.expasy.org/protparam/).

\section{RNA extraction and analysis of Sjpcdp10 mRNA expression by real-time quantitative PCR (qPCR)}

Total RNA was extracted from schistosomula using TRIzol reagent (Invitrogen, Carlsbad, CA, USA), according to manufacturer instructions. After removing the genomic DNA with RNase-free DNase (Takara Bio, Shiga, Japan), cDNA was synthesized using the RevertAid First Strand cDNA synthesis kit (Fermentas, Vilnius, Lithuania), according to the standard protocol. All reactions were performed with a Bio-Rad CFX96 detection system (Bio-Rad, Hercules, CA, USA). Primers used for real-time qPCR were designed with Beacon Designer version 8.14 software (PREMIER Biosoft, Palo Alto, CA, USA) and synthesized by Sunny Co., Ltd. Primer sequences are shown in Additional file 1: Table S1. Primers were used to amplify a 142-bp fragment of Sjpcdp10 (GenBank: FN326945.1), and another pair of primers was used for amplifying a 213-bp fragment of the $\alpha$-tubulin gene of S. japonicum (GenBank: AY815746.1) as an internal control [16]. Melting curve analyses of the specific PCR products were performed, and each experiment was performed in triplicate. Bio-Rad CFX Manager 3.1 software (Bio-Rad) was used to analyze $\operatorname{Sjpcdp10}$ transcript levels relative to those of $\alpha$-tubulin, according to the $2^{-\Delta \Delta \mathrm{Ct}}$ method [17] .

\section{Immunolocalization of rSjPCDP10}

The recombinant pET28a (+)-SjPCDP10 plasmids were prepared using the AxyPrep plasmid miniprep kit (Axygen, Hangzhou, China) and then transformed into competent E. coli BL21 (DE3) cells (Novagen, Madison, USA) to express the $\mathrm{r} S j \mathrm{PCDP} 10$ protein. A large amount of $\mathrm{rSjPCDP} 10$ protein was expressed at $37{ }^{\circ} \mathrm{C}$ for $3 \mathrm{~h}$ following induction with $0.5 \mathrm{mM}$ isopropyl- $\beta$ thiogalactopyranoside and purified using Ni-NTA agarose (QIAGEN, Hilden, Germany) affinity purification according to manufacturer instructions. Purified protein was quantified using the $\mathrm{BCA}$ protein assay kit (Beyotime, Shanghai, China) according to manufacturer instructions and administered to New Zealand male rabbits in the neck and back by multipoint subcutaneous injection at a dose of $1 \mathrm{mg} / \mathrm{kg} \mathrm{rSjPCDP} 10$ emulsified with adjuvant. The immunization was performed three times at time intervals of 2 weeks. Ear venous blood was exsanguinated from the rabbits and used to assess the antibody titer by enzyme-linked immunosorbent assay. Anti-rSjPCDP10 sera were collected 2 weeks after the final immunization. Negative control sera were collected from rabbits immunized with $8 \mathrm{M}$ urea emulsified with an adjuvant.

All parasites were fixed with $4 \%$ paraformaldehyde overnight at room temperature $\left(20-25{ }^{\circ} \mathrm{C}\right)$ and then embedded in paraffin. Immunolocalization of SjPCDP10 was assessed by an immunohistochemical method previously described [18]. Rabbit-anti-rSjPCDP10 serum (1:100 dilution; sera prepared as described) was used as the primary antibody, and Cy3-conjugated goat antirabbit (red fluorescence) was used as the secondary antibody (1:3000 dilution; KPL, Gaithersburg, MD, USA). Cell nuclei were stained blue fluorescence using 4',6diamidino-2-phenylindole dye solution for $5 \mathrm{~min}$ and washed with PBS. After fixing with a cover glass containing anti-fade mounting medium (Beyotime), slides were observed under a fluorescence microscope (OLYMPUS, Tokyo, Japan), followed by semi-quantitative analysis by measuring the optical density value of SjPCDP10 protein distribution area using IPP6.0 software (Media Cybernetics, Rockville, USA).

\section{Western blot analysis}

Total soluble protein was obtained from worms at different stages according to a method described previously [19]. Purified $\mathrm{rSjPCDP10}(4 \mu \mathrm{g})$ and total soluble protein $(20 \mu \mathrm{g})$ of schistosomula were subjected to sodium dodecyl sulfate-polyacrylamide gel electrophoresis and then transferred electrophoretically onto a $0.45-\mu \mathrm{m}$ pore nitrocellulose membrane (Whatman; GE Healthcare, Little Chalfont, UK) at $80 \mathrm{~V}$ for $70 \mathrm{~min}$. Membranes were blocked with $5 \%(w / v)$ non-fat dried milk in trisbuffered saline with $0.05 \%(v / v)$ Tween-20 (TBS/T) for $1 \mathrm{~h}$ at $37^{\circ} \mathrm{C}$. Membranes were then incubated overnight with anti-rSjPCDP10 rabbit serum (1: 1000) and anti- $\alpha$ tubulin rabbit polyclonal antibody (1:3000 dilution; TDYBIO, Beijing, China) at $4{ }^{\circ} \mathrm{C}$. After washing with TBS/T every 5 min for four rounds, membranes were incubated with horseradish peroxidase-conjugated goat anti-rabbit secondary antibody (1:5000 dilution; KPL) for $2 \mathrm{~h}$ at $37^{\circ} \mathrm{C}$. The membranes were then washed with TBS/T every 5 min for three rounds and visualized using enhanced chemiluminescence reagent (ASPEN, Wuhan, China), according to manufacturer instruction. 


\section{RNA-mediated Sjpcdp10 knockdown}

Sjpcdp10-specific dsRNA and irrelevant enhanced green fluorescent protein (egfp) dsRNA (negative control) were synthesized in vitro with the T7 RiboMAX expression RNAi system (Promega, Durham, NC, USA) according to manufacturer instructions. Primers tagged with T7 RNA polymerase promoter sequences at both ends (Additional file 2: Table S2) were designed with the help of the online IDT RNAi Design Tool (https://sg.idtdna.$\mathrm{com} /$ site/order/designtool/index/DSIRNA_CUSTOM) to amplify a 560-bp DNA product from Sjpcdp10 cDNA by PCR. Primers for egfp were the same as reported by Liu et al. [18] and were used to amplify a 678-bp DNA product from pEGFP-N1 plasmids by PCR. dsRNA was prepared by DNA transcription in vitro and stored at $-20{ }^{\circ} \mathrm{C}$ after purification.

Cercariae were collected as described, mechanically transformed into schistosomula by syringe passage under sterile conditions [10], and cultured in vitro with "841" medium [a mixture of RPMI1640 (Gibco; Thermo Fisher Scientific, Waltham, MA, USA), 10\% fetal bovine serum (Gibco), $1 \mu \mathrm{M}$ 5-hydroxytryptamine, $1 \mu \mathrm{M}$ L-hydrocortisone, $0.5 \mu \mathrm{M}$ hypoxanthine, $0.2 \mathrm{U} / \mathrm{ml}$ insulin, $100 \mathrm{U} / \mathrm{ml}$ penicillin, $100 \mu \mathrm{g} / \mathrm{ml}$ streptomycin [10], and a concentration gradient of $0.5,1,2,4,8,16,32$ or $64 \times 10^{-5} \mathrm{mg} / \mathrm{ml} \mathrm{Sjpcdp10}$ dsRNA, with no dsRNA as a blank control and $4 \times 10^{-5} \mathrm{mg} / \mathrm{mL}$ egfp dsRNA (same as the optimal concentration of Sjpcdp10 dsRNA) used as a negative control. Schistosomula were cultured at $37{ }^{\circ} \mathrm{C}$ in an incubator with $5 \% \mathrm{CO}_{2}$ for 1 to 7 days, with the medium and dsRNA replaced every other day.

To monitor gene expression and dsRNA concentrations at various time points following dsRNA soaking, real-time qPCR was performed using the conditions described. To measure protein levels after RNAi, western blot analysis was performed after RNAi under optimal conditions. To compare the parasite sizes of the control and RNAi groups, images were obtained under a stereomicroscope (Olympus SZX7; OLYMPUS), and the lengths (L) and widths (W) of schistosomula were analyzed by Image-pro plus 6.0 software (Media Cybernetics), followed by L: W ratio calculations [10]. Routine dehydration, desiccation and spray gold were performed, and SEM was used to observe the surface morphology using a VEGA 3 LMU scanning electron microscope (TESCAN, Brno, Czech Republic). TEM specimens were fixed, dehydrated, embedded and then cut into ultrathin sections. Ultrastructural alterations were observed by H-7700 transmission electron microscopy (TEM; Hitachi, Tokyo, Japan). The terminal deoxynucleotidyl transferase dUTP nick-end labelling (TUNEL) method (in situ cell death detection kit; Roche, Basel, Sweden) was used to examine the occurrence of apoptosis in schistosomula after Sjpcdp10 knockdown, and images were obtained under an IX51 inverted microscope (OLYMPUS).

\section{Statistical analysis}

All data are expressed as the mean \pm standard deviation. Statistical analysis for quantitative RT-PCR and antibodies was performed by analysis of variance. A $P<0.05$ was considered significant. All RNAi results were compared with those of the blank control group.

\section{Results}

Molecular cloning and sequence analysis of SjPCDP10 Sjpcdp10 cDNA (GenBank: FN326945.1) contains an open reading frame of 651-bp, encoding a putative 216aa protein with a predicted molecular weight of $26 \mathrm{kDa}$ and a calculated isoelectric point of 9.11. Bioinformatics analysis revealed that SjPCDP10 is an unstable (instability index: 40.46) and hydrophilic (GRAVY: -0.269) protein. No typical signal-peptide sequence, a transmembrane region, or $\mathrm{N}$-glycosylation sites were predicted according to SignalP 4.1 (http://www.cbs.dtu.dk/services/ SignalP/), TMHMM version. 2.0 (http://www.cbs.dtu.dk/ services/TMHMM/), and NetNGlyc 1.0 (www.cbs.dtu.dk/ services/NetNGlyc/).

ClustalX 2.0 alignment of the SjPCDP10 sequence with PCDP10 homologs from trematodes and other species revealed that SjPCDP10 shared the highest similarity with PCDP10 proteins from Schistosoma mansoni (96\%) and Schistosoma haematobium (93\%), followed by lower levels of similarity with those from Clonorchis sinensis (53\%), Opisthorchis viverrini (53\%), Homo sapiens (42\%) and Mus musculus (42\%) (Fig. 1a). Sequences of PCDP10 orthologues in species from several taxa were used to build a phylogenetic tree using the neighbour-joining method (Fig. 1b) [20]. SjPCDP10 clustered with the PCDP10 proteins of trematodes (S. mansoni, S. haematobium, C. sinensis, Echinostoma caproni, Trichobilharzia regenti and O. viverrini), forming a common clade.

\section{Sjpcdp10 mRNA expression at different developmental stages}

The transcript levels of Sjpcdp10 mRNA at different schistosomula stages were determined by real-time qPCR. As shown in Fig. 2, Sjpcdp10 mRNA was expressed at all investigated developmental stages. Compared with levels expressed in skin-stage schistosomula, the Sjpcdp 10 expression levels were higher in 14-, 18-, and 21-day-old schistosomula $\left(F_{(5,12)}=8.69, \quad P<0.05\right)$. There was no significant difference among the other groups.

\section{rSjPCDP10 immunolocalization}

An immunolocalization assay was performed to determine tissue localization of the SjPCDP10 protein in schistosomula and using negative control serum as the primary antibody for the control group. As shown in Fig. 3, SjPCDP10 was mainly distributed in the teguments of schistosomula in all investigated stages and 


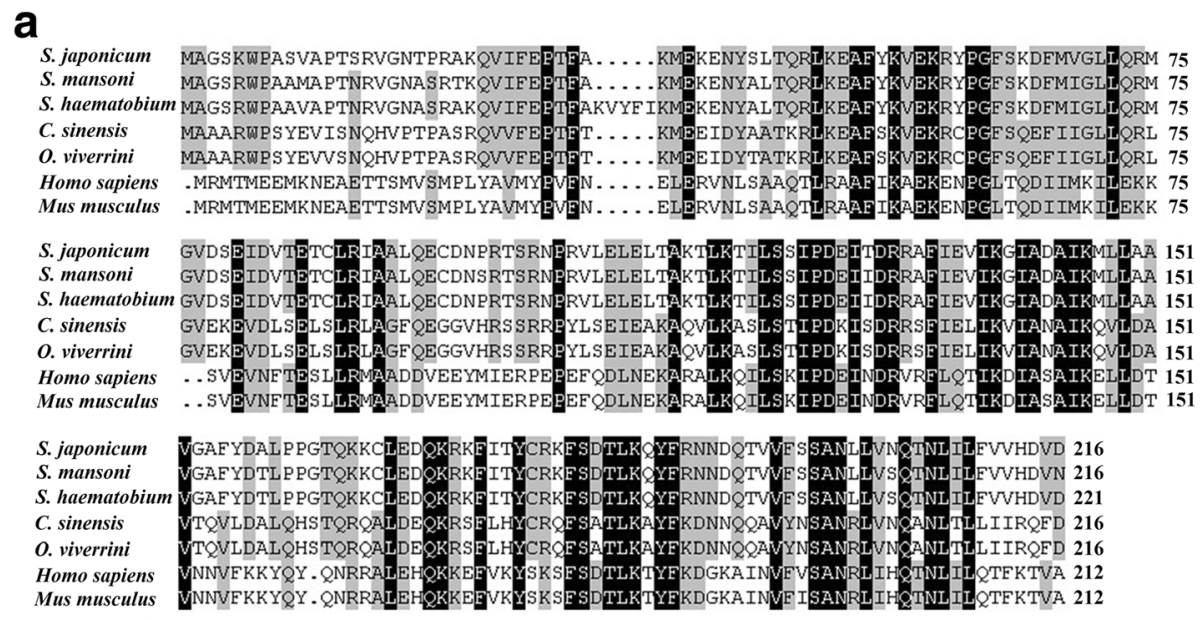

b

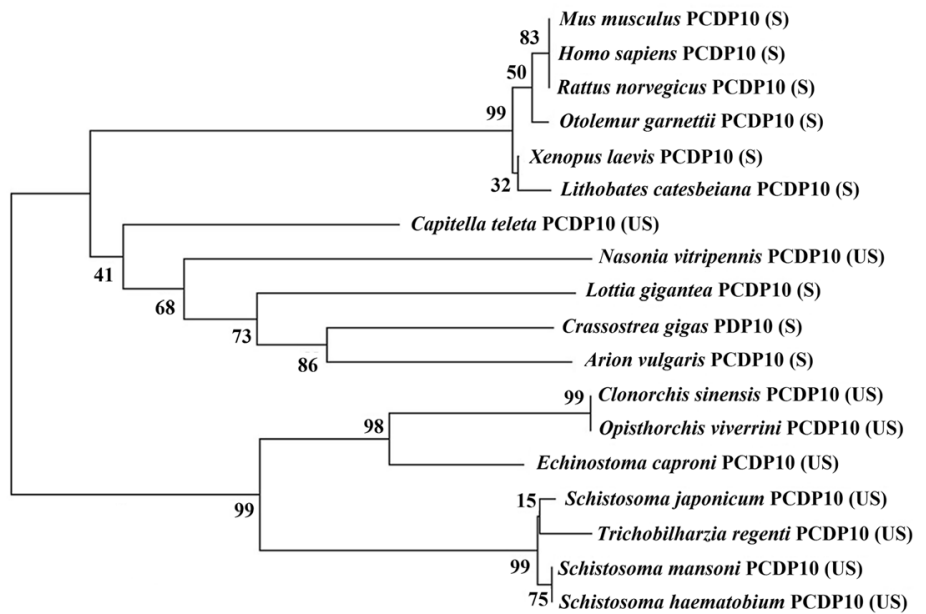

Fig. 1 Bioinformatics analysis of Schistosoma japonicum PCDP10 (SjPCDP10). a Amino acid sequence alignment of PCDP10 from S. japonicum. Black background indicates $100 \%$ identity in all species, dark grey indicates $\geq 75 \%$ identity, and light grey indicates $\geq 50 \%$ identity. $\mathbf{b}$ Phylogenetic tree of SjPCDP10 and homologs from other species generated using the neighbour-joining method. The S.PPCDP10 protein was most closely related to PCDP10 proteins of Schistosoma mansoni and Schistosoma haematobium and showed low homology with those from other species. UniProtKB accession numbers are as follows: S. japonicum (Q5D8L4), S. mansoni (C4Q5Z1), S. haematobium (A0A095APY2), Clonorchis sinensis (H2KTN8), Opisthorchis viverrini (A0A074ZX12), Trichobilharzia regent (A0A183XOF3), Echinostoma caproni (A0A183AMZ2), Capitella teleta (R7TWK6), Caenorhabditis elegans (Q17958), Homo sapiens (Q9BUL8), Mus musculus (Q8VE70), Rattus norvegicus (Q6NX65), Otolemur garnettii (H0XA12), Lithobates catesbeiana (C1C3N3), Crassostrea gigas (K1PTV5), Strigamia maritima (T1JN96), Nasonia vitripennis (K7IPU5), Arion vulgaris (A0A0B6Y0C7), Lottia gigantea (V4B5U4), Zootermopsis nevadensis (A0A067R7A2), and Xenopus laevis (Q8AVR4). Abbreviations: S, stable; US, unstable

part of the parenchymal areas of 14-, 18-, and 21-dayold schistosomula. The area of each positive region was calculated using IPP 6.0 software, revealing that SjPCDP10 expression levels were lower in skin-stage and lung-stage schistosomula and higher in 21-day-old schistosomula (Additional file 3: Table S3), which was consistent with qPCR (Fig. 3) results and western blot result (Additional file 4: Figure S1).

\section{Preparation of dsRNA and analysis of optimal RNAi conditions by qPCR and western blot}

dsRNA was synthesized using the Sjpcdp10 RNAi target sequence as a template and then added to the medium of schistosomula cultured in vitro to screen the optimal RNAi condition. RNAi efficiency was compared against the transcription level of Sjpcdp10 under different interference conditions. As shown in Fig. 4a, the size of Sjpcdp10 dsRNA was $\sim 560$-bp, which was consistent with the predicted result. Sjpcdp10 dsRNA was added to culture medium containing schistosomula cultured in vitro, followed by determination of the optimal RNAi conditions by qPCR. The optimal RNAi concentration of Sjpcdp10 dsRNA was $4 \times 10^{-5} \mathrm{mg} / \mathrm{ml}$, and the optimal RNAi duration was 6 days (Fig. 4b, c).

dsRNA was added to the medium of schistosomula cultured in vitro at the optimal condition. Western blot 


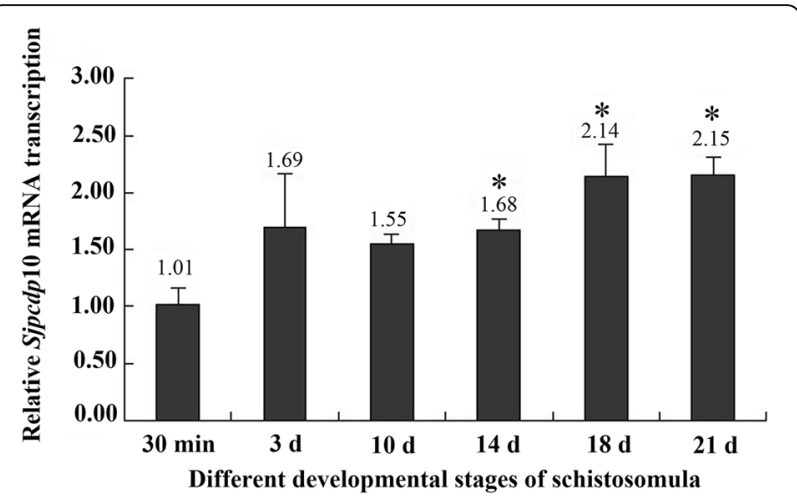

Fig. 2 Sjpcdp10 transcript levels in the schistosomulum stage of the Schistosoma japonicum life-cycle. Stages included skin-stage schistosomula (30 min), 3-day-old lung-stage schistosomula (3 d), 10-day-old liver-stage schistosomula (10 d), 14-day-old liver-stage schistosomula (14 d), 18-day-old liver-stage schistosomula (18 d), and 21-day-old liver-stage schistosomula (21 d). Sjpcdp10 transcript levels at the different schistosomula stage were compared by multiple comparisons. The expression of S. japonicum a-tubulin was used as an internal control. All experiments were performed in triplicate, and the figure above each bar is the mean of each group. Asterisks denote significant differences $(P<0.05)$ results were analyzed using AlphaEaseFC software through the grey value ratio associated with SjPCDP10: $\alpha$-tubulin, which represented the relative SjPCDP10 protein expression in different RNAi group. Compared with the control group (Fig. 5), SjPCDP10 expression of the Sjpcdp10 dsRNA treated group decreased by $60 \%$ relative to that observed in the control group. Compared with the egfp negative control group, SjPCDP10 expression decreased by $25 \%$. These results showed that the RNAi experiment successfully achieved gene knockdown.

\section{Morphological changes in schistosomula after RNAi}

The sizes of the schistosomula, and their ultrastructure was studied by SEM and TEM. The length, width, area, and volume of Sjpcdp10-knockdown schistosomula were significantly lower than those of the blank control group (Table 1). Based on SEM observations, schistosomula from the blank control (Fig. 6a, a') or negative control (egfp RNAi) groups (Fig. 6b, b') both exhibited plump bodies with multiple spines distributed irregularly, as well as numerous sensory papillae on the body surface (Fig. 6a, a', b, b'). By contrast, the surfaces of schistosomula subjected to Sjpcdp10 knockdown were severely damaged, with tegument structure profoundly disordered,
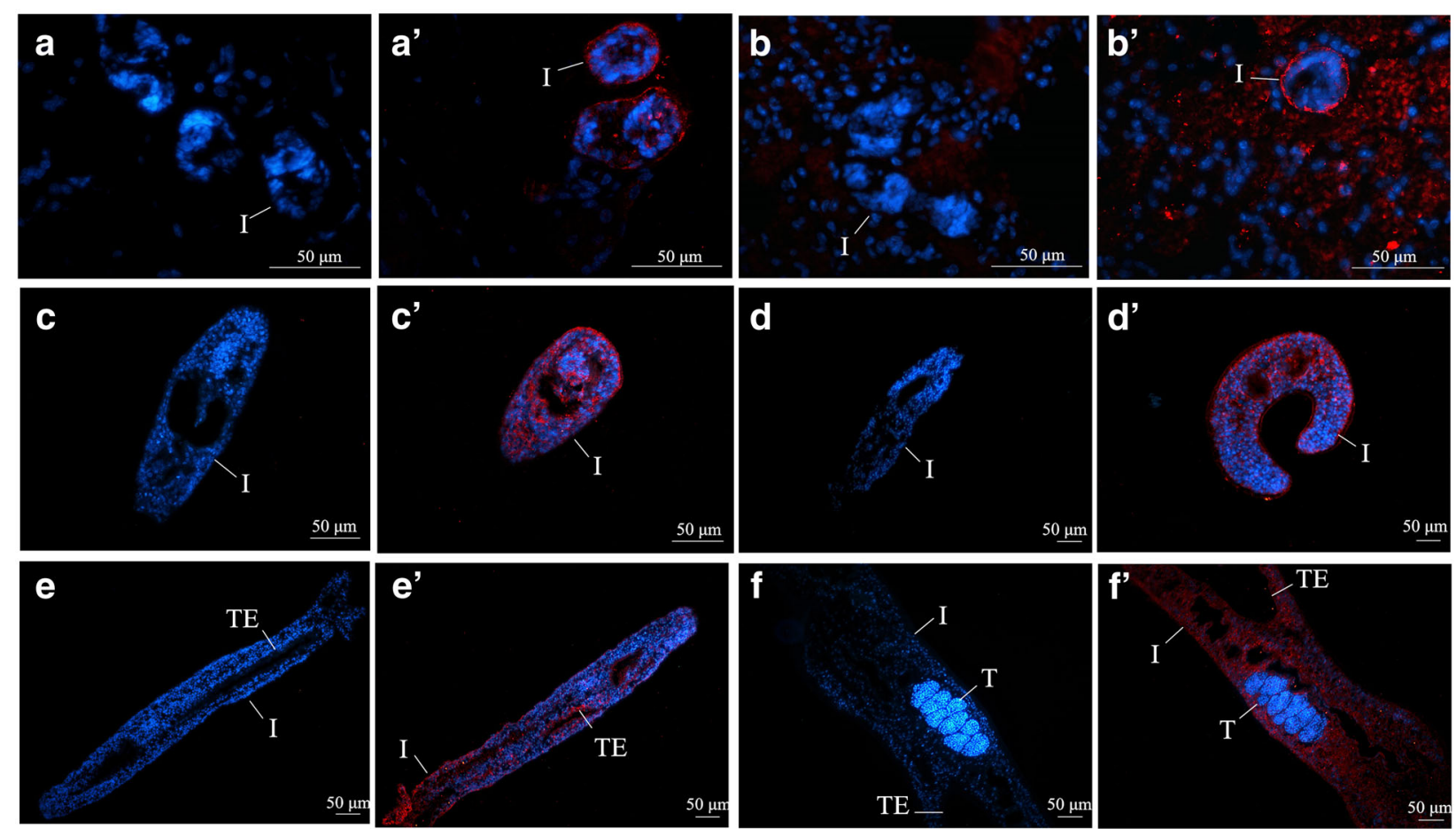

Fig. 3 Immunolocalization of SJPCDP10 in different tissues of Schistosoma japonicum. a-f Skin-stage, lung-stage, and 10-, 14-, 18-, and 21-day-old liver-stage schistosomula were probed with negative rabbit sera. a'-f' Skin-stage, lung-stage, and 10-, 14-, 18-, and 21-day-old liver-stage schistosomula were probed with anti-rS.PCDP10 rabbit sera. S.PCDP10 protein was found to be present in the different developmental stages of schistosomula. Red fluorescence indicates areas positive for the SJPCDP10 protein. Blue fluorescence represents the cell nucleus. Images $\mathbf{a}$ and $\mathbf{a}$ ' were obtained from the skin of mice infected with cercaria. Images $\mathbf{b}$ and $\mathbf{b}^{\prime}$ were obtained from the lung of mice infected with cercaria. Abbreviations: I, integument; TE, intestinal epithelium; T, testis 


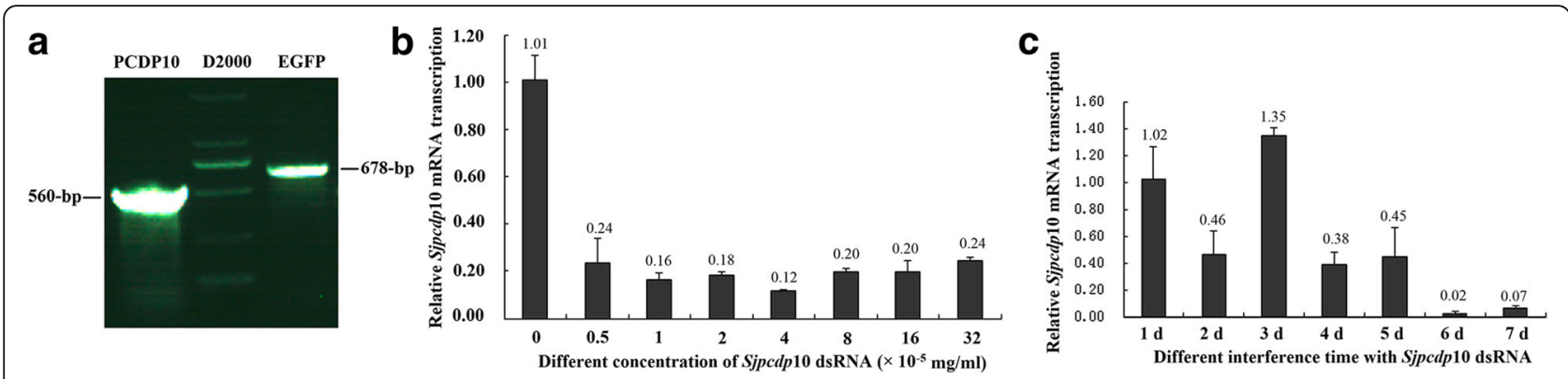

Fig. 4 Preparation of Sjpcdp10 dsRNA and analysis of the optimal RNAi conditions. a Agarose gel electrophoresis of Sjpcdp10 dsRNA. Lane PCDP10: Sjpcdp10 dsRNA; Lane D2000: markers; Lane EGFP: egfp dsRNA (negative control dsRNA). b Analysis of the optimal concentration of Sjpcdp10 dsRNA at the optimal RNAi time (6 days). Sjpcdp10 dsRNA concentrations: 0, 0.5, 1, 2, 4, 8, 16, 32, $64 \times 10^{-5} \mathrm{mg} / \mathrm{ml}$. c Analysis of the optimal incubation/soaking time with the optimal concentration of Sjpcdp10 dsRNA ( $\left.4 \times 10^{-5} \mathrm{mg} / \mathrm{ml}\right)$. RNAi incubations times with Sjpcdp10 dsRNA: $1 \mathrm{~d}, 2 \mathrm{~d}, 3 \mathrm{~d}, 4 \mathrm{~d}, 5 \mathrm{~d}, 6 \mathrm{~d}, 7 \mathrm{~d}$

fewer spines and no sensory papillae present at the surface (Fig. 6c, c'). According to TEM, the tegument structures of schistosomula of the blank control (Fig. 6d, d') or negative control (egfp RNAi) groups (Fig. 6e, e') were intact, whereas the teguments of the Sjpcdp10-knockdown group were partially invaginated, interrupted, or bloated, and the subtegumental layer appeared reduced in thickness (Additional file 5: Figure S2) as compared with the two control group (Fig. 6f, $\mathrm{f}^{\prime}$ ). Early apoptosis was observed in all groups according to the appearance of chromatin at the nuclear membrane and shrinkage of the nuclear membrane (Fig. 6f, $\mathrm{f}^{\prime}$ ).

\section{Detection of apoptosis in schistosomula after RNAi by TUNEL assay}

Schistosomula were collected after RNAi, and paraffin sections were prepared. Rates of apoptosis were then determined by TUNEL assay, revealing that the rate of TUNEL-positive cells in the Sjpcdp10-knockdown group was $22.77 \%$ higher $\left(F_{(3,8)}=168.07, P<0.05\right)$ than that of the control group (Fig. 7).

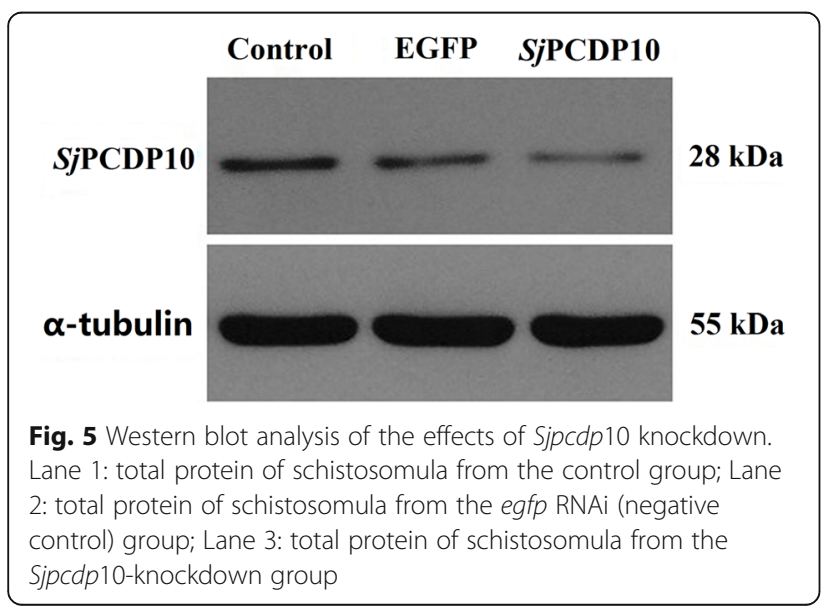

\section{Discussion}

PCDP10 is an evolutionarily ancient, highly conserved gene with homologs in many different organisms, including nematodes, Drosophila, and zebrafish [14]. In this study, it was shown that SjPCDP10 was highly similar to S. mansoni PCDP10 and S. haematobium PCDP10, with lower rates of similarity to other species, although a high degree of conservation was observed (Fig. 1a). The phylogenetic tree showed that the predicted PCDP10 proteins of trematodes and nematodes are unstable, whereas those of vertebrates are stable (Fig. 1b). Here, rSjPCDP10 prepared using a prokaryotic expression system was unstable, consistent with the predicted results, and the purified $\mathrm{rSjPCDP} 10$ protein was easily degraded into two small molecules of $15 \mathrm{kDa}$ and $10 \mathrm{kDa}$. Therefore, $\mathrm{rSjPCDP} 10$ must be freshly prepared before use in protein-immunization experiments. Currently, there are no reports concerning PCDP10 stability in other species, therefore, this requires additional data screening and experimental proof.

In humans, PCDP10 is widely expressed in various tissues, such as the placenta, liver, and kidney [12], as well as the uterus, ovary, lung, stomach, and small intestine, suggesting that PCDP10 might have a more generalized biological function [13]. In the present study, Sjpcdp10 mRNA was expressed in all investigated schistosomula developmental stages, with Sjpcdp10 expression level elevated along with schistosomula growth and development (Fig. 2). Similar trends were observed for SjPCDP10 protein expression at different schistosomula stages according to western blot analysis (Additional file 4: Figure S1). This indicated that the demand for SjPCDP10 increased along with continuous schistosomula growth and development, suggesting that SjPCDP10 might be involved in schistosomula development. Immunohistochemical analysis revealed that SjPCDP10 was mainly distributed in the teguments and the parenchymal areas of schistosomula at different 
Table 1 Morphological changes of the schistosomula after interference

\begin{tabular}{lllllll}
\hline Group & RNAi time (days) & No. of worms & $\begin{array}{l}\text { Mean length } \pm \\
\text { SD }(\mu \mathrm{m})\end{array}$ & $\begin{array}{l}\text { Mean width } \pm \\
\mathrm{SD}(\mu \mathrm{m})\end{array}$ & $\begin{array}{l}\text { Mean volume } \pm \\
\mathrm{SD}\left(\times 10^{4} \mu \mathrm{m}^{3}\right)\end{array}$ & $\begin{array}{l}\text { Mean area } \pm \\
\mathrm{SD}\left(\times 10^{4} \mu \mathrm{m}^{2}\right)\end{array}$ \\
\hline Blank control & 6 & 90 & $129.88 \pm 17.10$ & $66.19 \pm 10.57$ & $23.00 \pm 7.72$ & $3.41 \pm 0.79$ \\
EGFP & 6 & 77 & $127.31 \pm 14.05$ & $62.21 \pm 8.59$ & $20.63 \pm 5.56$ & $3.10 \pm 0.58$ \\
SjPCDP10 & 6 & 115 & $122.74 \pm 14.20^{*}$ & $56.93 \pm 6.45^{*}$ & $17.44 \pm 4.03^{*}$ & $2.70 \pm 0.41^{*}$ \\
\hline
\end{tabular}
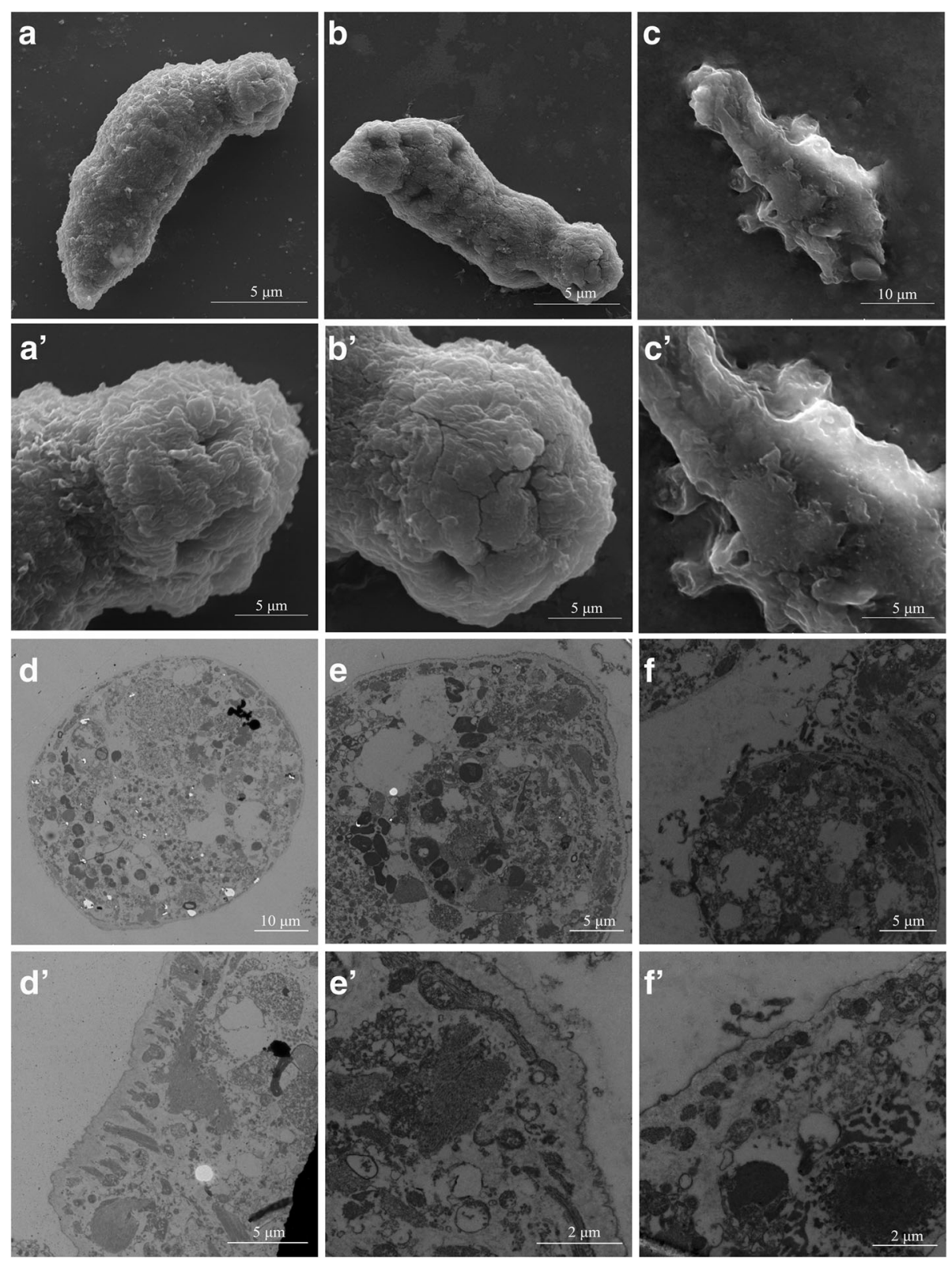

Fig. 6 Ultrastructural changes in schistosomula after Sjpcdp10-knockdown experiments and analysis by electron microscopy. SEM analysis of morphological changes of the surface of blank control (a, $\left.\mathbf{a}^{\prime}\right)$, egfp negative control $\left(\mathbf{b}, \mathbf{b}^{\prime}\right)$ and Sjpcdp10-knockdown schistosomes (c, $\left.\mathbf{c}^{\prime}\right)$. TEM analysis of the morphological changes in the cell and tissue of blank control (d, $\left.\mathbf{d}^{\prime}\right)$, egfp negative control (e, $\left.\mathbf{e}^{\prime}\right)$ and Sjpcdp10-knockdown schistosomes (f, $\left.\mathbf{f}^{\prime}\right)$. SEM and TEM results of Sjpcdp10-knockdown schistosomes compared with those of the blank control and egfp negative control 


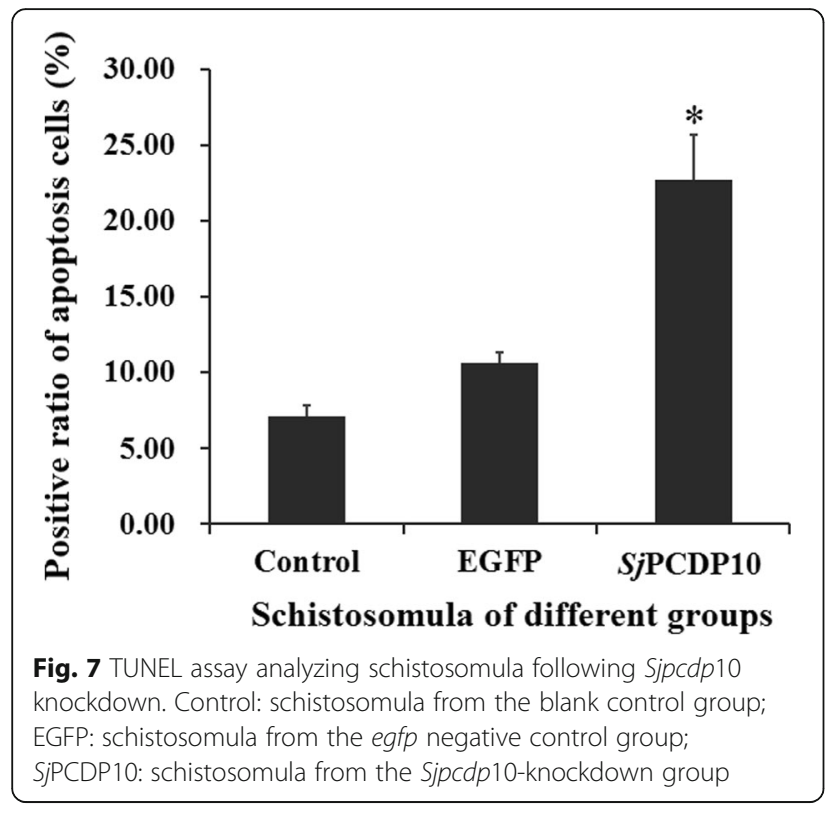

developmental time points (Fig. 3). The predominant occurrence of SjPCDP10 in the tegument of schistosomula suggested that SjPCDP10 might be a potential functional molecule on the surface and that its function might be related to the tegument/subtegument.

RNAi has been widely used to study the functions of $S$. mansoni and S. japonicum at different growth stages $[8,21-24]$. The optimal RNAi conditions for knocking down Sjpcdp10 in S. japonicum schistosomula were systematically determined according to various parameters, including dsRNA concentrations and soaking periods. Results based on qPCR and western blot revealed that the optimal dsRNA concentration was $4 \times 10^{-5} \mathrm{mg} / \mathrm{ml}$, and the optimal incubation period (soaking time) was 6 days (Fig. 4). Under these conditions, the sizes of treated schistosomula were significantly lower than those in the untreated control group (Table 1). Moreover, the surfaces of treated schistosomula were seriously damaged, with invaginations, interruptions, and fewer spines, as well as sensory papillae on the surface (Fig. 6). Programmed cell death (PCD) is a physiological cell death mechanism that can have a positive effect on organism growth and development. In some cases, PCD is fundamentally important for normal growth, as well as to overcome adverse environments $[25,26]$. The tegument of schistosomes represents the first physical line of defence against the host environment and the main site of host-parasite interaction. The tegument of schistosomes functions in areas that include nutrient uptake, immune evasion, excretion, osmotic pressure regulation, and signal transduction [27, 28]. Due to the active growth and metabolism of $S$. japonicum, the tegument is constantly being shed, and damaged teguments must be quickly repaired $[29,30]$. Although most schistosomula of each group could live well in the RNAi experiment (Additional file 6: Figure S3), serious damage was observed in the teguments of schistosomula following Sjpcdp10 knockdown (Fig. 6), suggesting that SjPCDP10 might affect tegument development.

In humans, PCDP10 is an apoptosis-related gene [25]. When PCDP10 was added to the cell culture medium of a human embryonic kidney cell line 293, the number of apoptotic cells decreased along with increasing protein dosage [12]. Moreover, in some studies involving increased apoptosis induced by certain diseases, PCDP10 expression was also upregulated to inhibit apoptosis [31, 32]. In this study, the rate of apoptosis in the Sjpcdp10knockdown group increased significantly as compared with that in the blank control group (Fig. 7), which corresponded to the reported role of Homo sapiens PCDP10. This suggested that SjPCDP10 might be involved in S. japonicum growth and development, as well as apoptosis-related processes, which include tegumental repair and turnover processes.

\section{Conclusions}

In summary, the findings presented here demonstrated that SjPCDP10 was abundantly expressed in the schistosomulum tegument and parenchymal areas, and knockdown experiments showed that SjPCDP10 affected schistosomula growth and development, especially the development of the tegument. The mechanism by which SjPCDP10 influences schistosomula growth and development might involve the regulation of cell apoptosis. Our findings showed that SjPCDP10 plays an important role in schistosomula growth and development.

\section{Additional files}

Additional file 1: Table S1. Primer sequences for real-time $\mathrm{QPCR}$. (XLSX $10 \mathrm{~kb}$ )

Additional file 2: Table S2. Primer sequences for preparing dsRNA templates. Underlined sequences indicate the $\mathrm{T} 7$ promoter recognition site. (XLSX 9 kb)

Additional file 3: Table S3. Semi-quantitative analysis of S.PCDP10 through the results of immunofluorescence. Stages included skin-stage schistosomula (30 min), 3-day-old lung-stage schistosomula (3 d), 10-day-old liver-stage schistosomula (10 d), 14-day-old liver-stage schistosomula (14 d), 18-day-old liver-stage schistosomula (18 d), and 21-day-old liver-stage schistosomula (21 d). (XLSX $10 \mathrm{~kb}$ )

Additional file 4: Figure S1. Western blot analysis of SjPCDP10 protein expression at different time points of the schistosomulum stage of the $S$. japonicum life-cycle. Stages included skin-stage schistosomula (30 min),

3-day-old lung-stage schistosomula (3 d), 10-day-old liver-stage schistosomula (10 d), 14-day-old liver-stage schistosomula (14 d), 18-day-old liver-stage schistosomula (18 d), and 21-day-old liver-stage schistosomula (21 d). The expression of S. japonicum a-tubulin was used as an internal control. (TIFF 157 kb)

Additional file 5: Figure S2. Thickness measurement of the schistosomula integument by IPP6.0 software after Sjpcdp10-knockdown. 
Control: schistosomula from the blank control group; EGFP: schistosomula from the egfp negative control group; SJPCDP10: schistosomula from the Sjpcdp10-knockdown group. (TIFF $28 \mathrm{~kb}$ )

Additional file 6: Figure S3. The vitality detection of schistosomulae after Sjpcdp10-knockdown at the optimal RNAi optimal condition. a the schistosomula of the blank control group; $\mathbf{b}$ the schistosomula of the egfp negative control group; $\mathbf{c}$ the schistosomula of the Sjpcdp10-knockdown group. Most schistosomula of each group could live well in the RNAi experiment. (TIFF $7854 \mathrm{~kb}$ )

\section{Abbreviations}

CDNA: Complementary DNA; EGFP: Enhanced green fluorescent protein; PCD: Programmed cell death; PCDP10: PCD protein 10; PCR: Polymerase chain reaction; qPCR: Quantitative real-time PCR; RNAi: RNA interference: rSjPCDP10: Recombinant SjPCDP10; RT-PCR: Reverse transcription PCR; SEM: Scanning electron microscopy; Sjpcdp10: S. japonicum pcdp10; TBS/ T: Tris-buffered saline with $0.05 \%(\mathrm{v} / \mathrm{v})$ Tween-20; TEM: Transmission electron microscopy; TUNEL: Terminal deoxynucleotidyl transferase dUTP nick-end labeling

\section{Acknowledgments}

The authors thank Xiao Xue Ma and Qian Gao for their initial involvement in the project. We are sincerely indebted to Rui Zhou for providing laboratory facilities. We thank Christoph G. Grevelding and Shu Hong Li for critical reading of the manuscript and additional advice.

\section{Funding}

This work was supported by National Natural Science Foundation of China (No. 81273010) and the scientific research subject of Health and Family Planning Commission of Hubei Province (No. WJ2017X30).

\section{Availability of data and materials}

The datasets supporting the conclusions of this article are included in the article and its additional files.

\section{Authors' contributions}

HFD and YRG formulated the study design. The main experiments were performed by YRG and WLH. The manuscript was prepared by YRG and CLT. All authors read and approved the final manuscript.

\section{Ethics approval}

The conduct and procedures involving animals were performed in strict accordance with the guidelines of the Association for Assessment and Accreditation of Laboratory Animal Care International. All the animal study protocols were approved by the Institutional Animal Care and Use Committee of Wuhan University.

\section{Consent for publication}

Not applicable.

\section{Competing interests}

The authors declare that they have no competing interests.

\section{Publisher's Note}

Springer Nature remains neutral with regard to jurisdictional claims in published maps and institutional affiliations.

\section{Author details}

'Hubei Province Key Laboratory of Allergy and Immunology, Department of Parasitology, Wuhan University School of Basic Medical Sciences, Wuhan, Hubei Province 430071, China. ${ }^{2}$ Department of Physiology, School of Basic Medicine Sciences, Hubei University of Science and Technology, Xianning, Hubei Province 437000, China. ${ }^{3}$ Medical Department, City College, Wuhan University of Science and Technology, Wuhan, Hubei Province 430083, China. ${ }^{4}$ Department of Clinical Laboratory, Wuchang Hospital, Wuhan 430063, China.
Received: 4 July 2017 Accepted: 10 January 2018

Published online: 18 January 2018

\section{References}

1. Fenwick A, Jourdan P. Schistosomiasis elimination by 2020 or 2030 ? Int J Parasitol. 2016;467:385-8

2. Lei ZL, Zhang LJ, Xu ZM, Dang H, Xu J, Lv S, et al. Endemic status of schistosomiasis in People's Republic of China in 2014. Zhongguo Xue Xi Chong Bing Fang Zhi Za Zhi. 2015;276:563-9. (In Chinese)

3. Song LG, Wu XY, Sacko M, Wu ZD. History of schistosomiasis epidemiology, current status, and challenges in China: on the road to schistosomiasis elimination. Parasitol Res. 2016;115(11):4071-81.

4. El Ridi $\mathrm{R}$, Tallima $\mathrm{H}$. Why the radiation-attenuated cercarial immunization studies failed to guide the road for an effective schistosomiasis vaccine: a review. J Adv Res. 2015;6(3):255-67.

5. Merrifield M, Hotez PJ, Beaumier CM, Gillespie P, Strych U, Hayward T, et al. Advancing a vaccine to prevent human schistosomiasis. Vaccine. 2016;34(26):2988-91.

6. Tebeje BM, Harvie M, You H, Loukas A, McManus DP. Schistosomiasis vaccines: where do we stand? Parasit Vectors. 2016:9:528.

7. Jones MK, Gobert GN, Zhang L, Sunderland P, McManus DP. The cytoskeleton and motor proteins of human schistosomes and their roles in surface maintenance and host-parasite interactions. BioEssays. 2004;26(7):752-65.

8. Correnti JM, Brindley PJ, Pearce EJ. Long-term suppression of cathepsin B levels by RNA interference retards schistosome growth. Mol Biochem Parasitol. 2005;143(2):209-15.

9. Nabhan JF, El-Shehabi F, Patocka N, Ribeiro P. The 265 proteasome in Schistosoma mansoni: bioinformatics analysis, developmental expression, and RNA interference (RNAi) studies. Exp Parasitol. 2007;117(3):337-47.

10. Ye Q, Zhu JY, Ming ZP, Zhao QP, Grevelding CG, Liu R, et al. Studies on the establishment of a co-culture system of lung stage Schistosoma japonicum with host cells. Parasitol Res. 2012;111(2):735-48.

11. Gao Y, Zhou X, Wang H, Liu R, Ye Q, Zhao Q, et al. Immunization with recombinant schistosome adenylate kinase 1 partially protects mice against Schistosoma japonicum infection. Parasitol Res. 2017;116(6):1665-74.

12. Wang $Y$, Liu H, Zhang $Y$, Ma D. cDNA cloning and expression of an apoptosis-related gene, humanTFAR15 gene. Sci China C Life Sci. 1999:42(3):323-9.

13. Bergametti F, Denier C, Labauge P, Arnoult M, Boetto S, Clanet M, et al. Mutations within the programmed cell death 10 gene cause cerebral cavernous malformations. Am J Hum Genet. 2005;76(1):42-51.

14. Ma X, Zhao H, Shan J, Long F, Chen $Y$, Chen $Y$, et al. PDCD10 interacts with Ste20-related kinase MST4 to promote cell growth and transformation via modulation of the ERK pathway. Mol Biol Cell. 2007;18(6):1965-78.

15. Ruppel A, Shi YE, Moloney NA. Schistosoma mansoni and S. japonicum: comparison of levels of ultraviolet irradiation for vaccination of mice with cercariae. Parasitology. 1990;101(1):23-6

16. Cheng G, Fu Z, Lin J, Shi Y, Zhou Y, Jin Y, et al. In vitro and in vivo evaluation of small interference RNA-mediated gynaecophoral canal protein silencing in Schistosoma japonicum. J Gene Med. 2009;11(5):412-21.

17. Pfaffl MW. A new mathematical model for relative quantification in real-time RT-PCR. Nucleic Acids Res. 2001;29(9):e45.

18. Liu $\mathrm{R}$, Zhao QP, Ye Q, Xiong $\mathrm{T}$, Tang $\mathrm{CL}$, Dong HF, et al. Cloning and characterization of a bone morphogenetic protein homologue of Schistosoma japonicum. Exp Parasitol. 2013;135(1):64-71.

19. Hong Y, Huang L, Yang J, Cao X, Han Q, Zhang M, et al. Cloning, expression and enzymatic characterization of 3-phosphoglycerate kinase from Schistosoma japonicum. Exp Parasitol. 2015;159:37-45.

20. Tamura K, Stecher G, Peterson D, Filipski A, Kumar S. MEGA6: molecular evolutionary genetics analysis version 6.0. Mol Biol Evol. 2013;30(12):2725-9.

21. Yang $Y$, Jin $Y$, Liu $P$, Shi $Y$, Cao $Y$, Liu J, et al. RNAi silencing of type $V$ collagen in Schistosoma japonicum affects parasite morphology, spawning, and hatching. Parasitol Res. 2012;111(3):1251-7.

22. Krautz-Peterson G, Simoes M, Faghiri Z, Ndegwa D, Oliveira G, Shoemaker $\mathrm{CB}$, et al. Suppressing glucose transporter gene expression in schistosomes impairs parasite feeding and decreases survival in the mammalian host. PLoS Pathog. 2010;6(6):e1000932.

23. Bhardwaj R, Krautz-Peterson G, Skelly PJ. Using RNA interference in Schistosoma mansoni. Methods Mol Biol. 2011;764:223-39. 
24. He Y, Cai G, Ni Y, Li Y, Zong H, He L. siRNA-mediated knockdown of two tyrosinase genes from Schistosoma japonicum cultured in vitro. Exp Parasitol. 2012;132(4):394-402.

25. Jaattela M. Programmed cell death: many ways for cells to die decently. Ann Med. 2002;34(6):480-8.

26. Latrasse D, Benhamed M, Bergounioux C, Raynaud C, Delarue M. Plant programmed cell death from a chromatin point of view. J Exp Bot. 2016; 67(20):5887-900

27. Fonseca $\subset$, Braz Figueiredo Carvalho G, Carvalho Alves C, de Melo TT. Schistosoma tegument proteins in vaccine and diagnosis development: an update. J Parasitol Res. 2012;2012:541268.

28. Van Hellemond JJ, Retra K, Brouwers JF, van Balkom BW, Yazdanbakhsh M, Shoemaker CB, et al. Functions of the tegument of schistosomes: clues from the proteome and lipidome. Int J Parasitol. 2006;36(6):691-9.

29. Liu F, Lu J, Hu W, Wang SY, Cui SJ, Chi M, et al. New perspectives on hostparasite interplay by comparative transcriptomic and proteomic analyses of Schistosoma japonicum. PLoS Pathog. 2006;2(4):e29.

30. Saunders N, Wilson RA, Coulson PS. The outer bilayer of the adult schistosome tegument surface has a low turnover rate in vitro and in vivo. Mol Biochem Parasitol. 1987:25(2):123-31.

31. Cardoso J, Boer J, Morreau H, Fodde R. Expression and genomic profiling of colorectal cancer. Biochim Biophys Acta. 2007;1775(1):103-37.

32. Xu Y, Kulkosky J, Acheampong E, Nunnari G, Sullivan J, Pomerantz RJ. HIV-1mediated apoptosis of neuronal cells: proximal molecular mechanisms of HIV-1-induced encephalopathy. Proc Natl Acad Sci U S A. 2004;101(18):7070-5.

\section{Submit your next manuscript to BioMed Central} and we will help you at every step:

- We accept pre-submission inquiries

- Our selector tool helps you to find the most relevant journal

- We provide round the clock customer support

- Convenient online submission

- Thorough peer review

- Inclusion in PubMed and all major indexing services

- Maximum visibility for your research

Submit your manuscript at www.biomedcentral.com/submit 